\title{
Phase of transmitted wave in dynamical theory and quasi-kinematical approximation
}

\author{
O. Yu. Gorobtsov ${ }^{1,2}$ and I. A. Vartanyants ${ }^{1,3, *}$ \\ ${ }^{1}$ Deutsches Elektronen-Synchrotron DESY, Notkestraße 85, D-22607 Hamburg, Germany \\ ${ }^{2}$ National Research Centre "Kurchatov Institute”, Akademika Kurchatova pl. 1, 123182 Moscow, Russia \\ ${ }^{3}$ National Research Nuclear University MEPhI (Moscow Engineering Physics Institute), Kashirskoe shosse 31, 115409 Moscow, Russia
}

(Received 17 November 2015; revised manuscript received 19 February 2016; published 23 May 2016)

\begin{abstract}
Variation of the phase of the beam transmitted through a crystalline material as a function of the rocking angle is a well-known dynamical effect in x-ray scattering. Unfortunately, it is not so easy to directly measure these phase variations in a conventional scattering experiment. It was recently suggested that the transmitted phase can be directly measured in ptychography experiments performed on nanocrystal samples. Results of such experiment for different crystal thickness, reflections, and incoming photon energies, in principle, can be fully described in the frame of dynamical theory. However, dynamical theory does not provide a simple analytical expression for the further analysis. Here we develop a quasi-kinematical theory approach that allows one to correctly describe the phase of the transmitted beam for the crystal thickness less than extinction length that is beyond applicability of the conventional kinematical theory.
\end{abstract}

DOI: 10.1103/PhysRevB.93.184107

\section{INTRODUCTION}

It is well known that the propagation of $\mathrm{x}$ rays through a slab of material results in the accumulation of a small phase shift due to refraction [1]. This phase shift plays an important role in different applications of $\mathrm{x}$ rays, despite $\mathrm{x}$ rays having a small index of refraction typically on the order of $10^{-5}$ to $10^{-7}$. For example, the focusing of $\mathrm{x}$ rays by compound refractive lenses [2] and phase contrast imaging [3] are based on refraction. It is also well known from the dynamical theory [4] that in the case of a perfect crystal, the phase of a diffracted wave in Bragg geometry changes by $\pi$ in the narrow angular range close to the Bragg angle. This is a manifestation of the $\mathrm{x}$-ray standing wave generated in a crystal by two coherent transmitted and diffracted waves [5-7]. It is interesting to note that the phase of the transmitted wave also has an additional dynamical correction close to the Bragg angle that depends on the rocking angle. Moreover, due to dynamical diffraction, this phase correction is different for $\pi$ and $\sigma$ polarization of $\mathrm{x}$ rays $[8,9]$. This effect is used nowadays to develop quarter- and half-wavelength phase shifters for hard $\mathrm{x}$ rays $[10,11]$ and to produce circular polarized radiation at third-generation synchrotron sources. Probably the most recent application of the transmitted beam phase in Bragg scattering geometry is the generation of self-seeded pulses of $\mathrm{x}$-ray free-electron lasers $[12,13]$.

It is not easy to measure the phase of the transmitted beam in the scattering experiment due to the fact that in a typical scattering experiment, it is intensity-the square modulus of a complex amplitude-that is measured on the detector. In Ref. [14], it was proposed to measure dynamical phase correction using interferometry measurements based on a Bonse-Hart interferometer [15]. By recombining two coherent beams passing through empty space and a crystal positioned close to the Bragg angle, an interference pattern was measured that could be well described in simulation by the presence of the phase of the transmitted beam. This was still indirect

\footnotetext{
*Corresponding author: ivan.vartaniants@desy.de
}

measurement of the phase. Recently developed coherent x-ray scattering methods, such as ptychography [16,17], can, in principle, measure the phase by reconstruction of the complex wave field. Ptychographic methods are highly sensitive to changes of phase and can be used to reconstruct the phase of the transmitted beam. Such experiment in which the phase of the transmitted beam in ptychographic measurements was determined was performed recently [18] (see layout of this experiment in Fig. 1).

In this experiment, two detectors were used, i.e., one in the diffraction and one in the transmission direction. Ptychographic measurements were performed both on empty membrane and $\mathrm{Au}$ crystalline nanoparticles $100 \mathrm{~nm}$ thick at different rocking angles of the sample. These measurements provided phase information of the transmitted beam that contained two components: the main one due to conventional refraction that does not depend on the rocking angle and another one that is much weaker and has an angular dependence on the rocking angle.

Though physical principles of generation of the transmitted wave phase are well understood and can be well simulated using dynamical theory [4], their solutions can be quite complicated in some special cases and often do not provide analytical results. The kinematical theory based on the assumption of a single scattering of the incident beam on the sample is comparatively easier to understand and interpret. However, kinematical theory does not describe variations of the phase close to the Bragg angle in a transmitted beam. To fill this gap, here we develop a quasi-kinematical theory that provides a simple analytical description for the phase of the transmitted beam.

Crystals with the thickness larger than the so-called extinction length [4] typically have to be described by the dynamical theory. At the same time, crystals with the thickness much smaller than this extinction length can be safely described by the kinematical theory [19]. The extinction length provides the typical crystal size for which multiple scattering effects, such as coupling between the transmitted and diffracted wave, become important. We will show that the quasi-kinematical approximation developed here gives correct results for the 


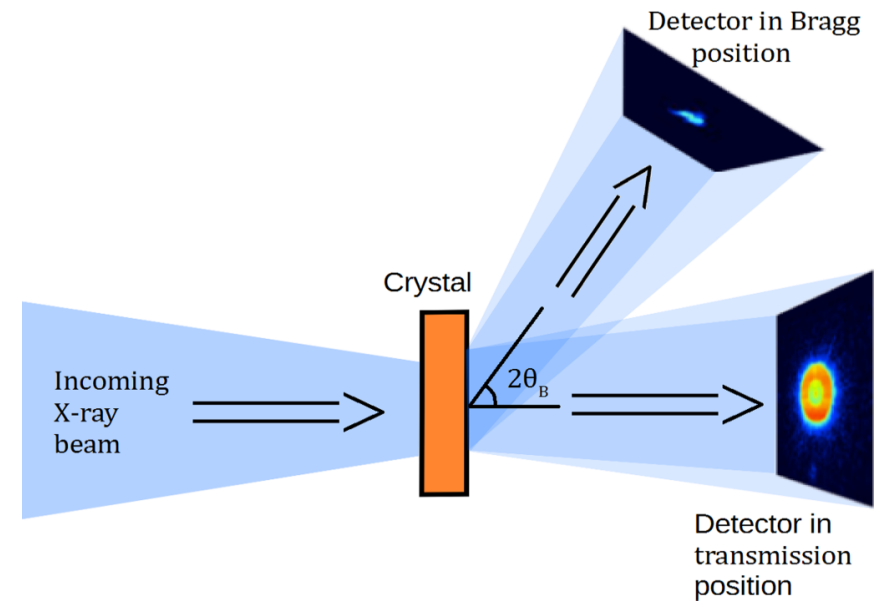

FIG. 1. Schematic layout of the ptychography experiment described in Ref. [18]. Intensities of the transmitted and diffracted beams are measured simultaneously by two detectors, while the crystal is rotated near the Bragg angle. Ptychographic measurements were performed on a Au 111 crystalline nanoparticle $100 \mathrm{~nm}$ thick. Reconstruction of the complex amplitude of the transmitted beam allowed one to observe phase variations as a function of the rocking angle.

transmitted phase for crystal thicknesses up to extinction lengths significantly beyond the conventional kinematical theory.

In this paper, we first revisit the concept of the phase of the transmitted beam for $\mathrm{x}$ rays. Using full dynamical theory, we show how the kinematical case can be generalized by including refraction and absorption effects. We present a general dynamical theory expression for the transmitted phase that is especially transparent in the case of a perfect crystal. We present simulations based on dynamical theory of the phase of the transmitted beam for different crystal types, reflection orders, and incoming photon energies and introduce a quasi-kinematical approximation to obtain an analytical solution for the dynamical phase contribution in the transmitted beam. We finalize our work with the summary and outlook section.

\section{GENERAL CONSIDERATIONS}

\section{A. Nonperiodic media}

We will first consider the case of scattering of $\mathrm{x}$ rays on nonperiodic media. In this case, refraction coefficient $n$ of matter for $\mathrm{x}$ rays is given by

$$
n=\sqrt{1+\chi_{0}} \simeq 1+\chi_{0} / 2
$$

where $\chi_{0}$ is the zeroth Fourier component of the susceptibility $\chi(\mathbf{r}, \omega)$ that is connected with the electron density $\rho$ by known expression [1] $\chi_{0}=-r_{e} \lambda^{2} \rho / \pi$, where $r_{e}$ is the classical electron radius and $\lambda$ is the $\mathrm{x}$-ray wavelength. Here we took into account that for $\mathrm{x}$ rays, $\chi_{0} \ll 1$. The $\mathrm{x}$-ray wave passing through a slab of material of thickness $d$ will get an additional phase shift,

$$
E_{\text {out }}(d)=E_{\text {in }}(z=0) e^{i \varphi(d)},
$$

where the total phase $\varphi(d)$ accumulated while passing the material is given by

$$
\varphi(d)=n k d / \gamma=\left(1+\chi_{0} / 2\right) k d / \gamma .
$$

Here, $k=\omega / c$ is the vacuum value of the incidence wave vector, $\omega$ is the frequency of $\mathrm{x}$ rays, $c$ is the speed of light, and $\gamma=\cos (\mathbf{n} \cdot \mathbf{k})$ is the direction cosine with $\mathbf{n}$ being the inward normal to the entrance surface of the material. We can see from that expression that the phase due to refraction in nonperiodic media is given by a simple expression,

$$
\varphi_{\mathrm{ref}}(d)=\chi_{0} k d /(2 \gamma) .
$$

This is a conventional phase shift due to refraction well known for electromagnetic waves which is proportional to an effective thickness of the material $t=d / \gamma$, the only difference for $\mathrm{x}$ rays is that it is negative since $\chi_{0}<0$.

\section{B. Periodic media}

For an X-ray wave passing through periodic media, the situation is similar to nonperiodic media in most cases, except incident angles close to the Bragg angle. As it follows from the dynamical theory [4], at these angles an additional dynamical correction $\delta n$ to refractive index $n$ will appear in expression (1). Contrary to expression (1), it will be dependent on the rocking angle $\Delta \theta$. Asymptotically, far from the exact Bragg condition, this refractive index correction can be expressed as $[4,20]$.

$$
\delta n \approx-\frac{C^{2} \chi_{h} \chi_{\bar{h}}}{4 \gamma_{0}\left(\Delta \theta-\theta_{\text {ref }}\right) \sin 2 \theta_{B}} .
$$

Here, $\chi_{h}$ and $\chi_{\bar{h}}$ are the Fourier components of the susceptibility of the $h$ and $\bar{h}$ reflections, respectively. They are connected with the Fourier components of the structure factor $F_{h}$ and $F_{\bar{h}}$ by well-known relations,

$$
\chi_{h}=-\Gamma F_{h}, \chi_{\bar{h}}=-\Gamma F_{\bar{h}},
$$

and parameter $\Gamma$ is given by $\Gamma=r_{e} \lambda^{2} / \pi V$, where $V$ is the volume of the unit cell. In expression (5), $C$ is the polarization coefficient equal to $C=1$ for $\sigma$ polarization and $C=\cos 2 \theta_{B}$ for $\pi$ polarization, $\Delta \theta=\theta-\theta_{B}$ is the angular deviation from the exact Bragg conditions, $\theta_{\text {ref }}$ is the angular correction due to refraction, and $\theta_{B}$ is the Bragg angle. We note that this expression is valid for both Bragg and Laue geometry. As was mentioned above, the range of validity of this expression is given by inequality

$$
|\Delta \theta| \gg\left|\chi_{h}\right| / \sin 2 \theta_{B} .
$$

It follows from expression (5) that the dynamical phase correction due to the coupling of transmitted and diffracted waves is proportional to the product of Fourier components of susceptibilities $\chi_{h} \chi_{\bar{h}}$ or structure factors $F_{h} F_{\bar{h}}$. This phase correction asymptotically decreases as $1 / \Delta \theta$ with the increase of the angular deviation from the Bragg angle and is proportional to the first power of a crystal thickness. Unfortunately, there is no simple relationship describing the phase of the transmitted beam for a more general case of an arbitrary thick crystal for the whole angular range near the Bragg angle, or a crystal with deformation field that could also change the values of the transmitted phase. In the following, we will analyze in detail the case of quasi-kinematical approximation when the 
phase due to dynamical scattering will be considered as a small perturbation to the phase $\varphi_{\text {ref }}(d)$ (4) due to refraction. The obtained analytical solution will be compared with full dynamical simulations and the range of validity of the quasikinematical approximation will be determined.

\section{DYNAMICAL THEORY APPROACH}

\section{A. Theory: General equations}

In the following, we will consider a plane $\mathrm{x}$-ray wave with a wave vector $\mathbf{k}$ incident on a single-crystal plate of thickness $d$. For generality, we will consider both Bragg and Laue diffraction geometries (see Fig. 2).

The wave field inside a crystal can be presented in the dynamical theory in two-wave approximation [4] as a coherent superposition of the transmitted $E_{0 s}(\mathbf{r})$ and diffracted $E_{h s}(\mathbf{r})$ waves,

$$
\mathbf{E}(\mathbf{r})=\sum_{s}\left[\mathbf{e}_{0 s} E_{0 s}(z) e^{i \mathbf{k}_{0} \mathbf{r}}+\mathbf{e}_{h s} E_{h s}(z) e^{i \mathbf{k}_{\mathbf{h}} \mathbf{r}}\right],
$$

where $\mathbf{e}_{0}$ and $\mathbf{e}_{h}$ are polarization unit vectors, $s$ is the polarization index, $\mathbf{k}_{\mathbf{0}}$ is the incident wave vector, and $\mathbf{k}_{\mathbf{h}}=$ $\mathbf{k}_{\mathbf{0}}+\mathbf{h}$ is the diffracted wave vector with $\mathbf{h}=2 \pi \mathbf{H}$ and $\mathbf{H}$ being the reciprocal space vector. Here we also assume that slowly varying amplitudes $E_{0 s}(z)$ and $E_{h s}(z)$ have only $z$ dependence.

Propagation of transmitted and diffracted amplitudes in a weakly deformed crystal can be described by the TakagiTaupin (TT) equations $[4,6,21,22]$

$$
\begin{aligned}
\frac{d E_{0 s}}{d z} & =\frac{i k}{2 \gamma_{0}}\left[\chi_{0} E_{0 s}(z)+\chi_{\bar{h}} C e^{i \mathbf{h u}(z)-W(z)} E_{h s}(z)\right], \\
\frac{d E_{h s}}{d z} & =\frac{i k}{2 \gamma_{h}}\left[\left(\chi_{0}-\alpha\right) E_{h s}(z)+\chi_{h} C e^{-i \mathbf{h u}(z)-W(z)} E_{0 s}(z)\right] .
\end{aligned}
$$

Here, $\mathbf{u}(z)$ and $W(z)$ are the strain field and static DebyeWaller factor [for a perfect crystal, $\mathbf{u}(z)=W(z)=0$ ], $\gamma_{0, h}=$ $\cos \left(\mathbf{n} \cdot \mathbf{k}_{0, h}\right)$ are the direction cosines, and $\mathbf{n}$ is the inward normal to the entrance surface of the crystal (see Fig. 2). For Bragg geometry of diffraction, $\gamma_{0}>0$ and $\gamma_{h}<0$, and for Laue diffraction, $\gamma_{0}>0$ and $\gamma_{h}>0$. Fourier components of the susceptibility are, in general, complex valued numbers $\chi_{h}=\chi_{h r}+i \chi_{h i}$. The parameter $\alpha$ characterizes the deviation of the incident wave vector $\mathbf{k}_{\mathbf{0}}$ from the Bragg condition $\alpha=$ $\left(k_{h}^{2}-k_{0}^{2}\right) / k_{0}^{2} \approx-2 \sin 2 \theta_{B} \Delta \theta$.

The boundary conditions in Bragg geometry are given by

$$
E_{0 s}(0)=E_{s}^{\text {in }}, \quad E_{h s}(d)=0,
$$

and in Laue geometry,

$$
E_{0 s}(0)=E_{s}^{\text {in }}, \quad E_{h s}(0)=0 .
$$

We will assume in the following a unit amplitude for the incoming beam, $E_{s}^{\text {in }}=1$.

The intensity of the diffracted beam, or reflectivity, is defined in Bragg geometry as

$$
p_{R}^{B}(\Delta \theta)=\frac{\left|\gamma_{h}\right|}{\gamma_{0}}\left|\frac{E_{h}(0, \Delta \theta)}{E_{0}(0, \Delta \theta)}\right|^{2},
$$

and in Laue geometry as

$$
p_{R}^{L}(\Delta \theta)=\frac{\gamma_{h}}{\gamma_{0}}\left|E_{h}(d, \Delta \theta)\right|^{2} .
$$

\section{B. Kinematical solution}

The kinematical solution [23] for the transmitted wave $E_{0}(z)$ can be obtained from Eq. (9a) by neglecting the coupling term with the diffracted amplitude $E_{h}(z)$. In this case, we obtain, from Eq. (9a),

$$
\frac{d E_{0 s}}{d z}=i \delta_{0} E_{0 s}(z) \text { where } \delta_{0}=\frac{k \chi_{0}}{2 \gamma_{0}} .
$$

Solution of this equation gives, for the $\mathrm{x}$-ray wave on the exit surface of the crystal,

$$
E_{0 s}^{\text {out }}(d)=e^{i \delta_{0} d}=\exp \left[i \varphi_{\text {ref }}(d)-\mu_{0} d / 2 \gamma_{0}\right],
$$

where

$$
\varphi_{\text {ref }}(d)=\operatorname{Re}\left[\delta_{0} d\right]=\frac{k \chi_{0 r}}{2 \gamma_{0}} d
$$

is the phase due to refraction [compare it with expression (4)] and $\mu_{0}=k \chi_{0 i}$ is the linear absorption coefficient.

Notice that transmitted wave $E_{0}(z)$ (15) determined in this way does not depend on the deviation angle from the exact Bragg condition $\Delta \theta$. It is only attenuated by absorption due to the imaginary part of the susceptibility $\chi_{0 i}$ and has a constant phase shift $\varphi_{\text {ref }}(d)$ due to the real part of the susceptibility $\chi_{0 r}$. By this treatment, we already go beyond the conventional kinematical theory that typically neglects these effects. However, we still neglected multiple scattering or dynamical effects that will be taken into account below.

\section{Dynamical solution}

An expression for the transmitted wave $E_{0}^{\text {out }}(d)$ on the exit surface of the crystal in the case of dynamical diffraction can be obtained as a formal solution of the TT equations (9) in the following form (for details, see [6]):

$$
E_{0}^{\mathrm{out}}(d, \Delta \theta)=\exp \left[i \varphi_{\mathrm{ref}}-\mu_{0} d / 2 \gamma_{0}+i \varphi_{\mathrm{dyn}}(d, \Delta \theta)\right],
$$

where $\varphi_{\text {dyn }}(d, \Delta \theta)$ is the phase contribution due to dynamical scattering given by

$$
\varphi_{\text {dyn }}(d, \Delta \theta)=-\frac{1}{L_{e x}} \operatorname{Re}\left[\int_{0}^{d} d z^{\prime} C_{1} R\left(z^{\prime}, \Delta \theta\right)\right] .
$$

Here, $R(z, \Delta \theta)$ is the scattering amplitude (for details, see Appendix A) defined as [6]

$$
R(z, \Delta \theta)=\frac{1}{\sqrt{\beta} Y}\left[\frac{E_{h}(z, \Delta \theta)}{E_{0}(z, \Delta \theta)}\right] e^{i \mathbf{h u}(z)} .
$$

In Eqs. (18) and (19), parameter $\beta=\gamma_{0} /\left|\gamma_{h}\right|$ for Bragg and $\beta=\gamma_{0} / \gamma_{h}$ for Laue geometries, $C_{1}=C(1-$ ip) $\exp [-W(z)]$ with $p=-X_{i} / X_{r}$, and parameter $Y=$ $\sqrt{\chi_{h} / \chi_{\bar{h}}}=|Y| \exp \left(i \Phi_{Y}\right)$. For a centrosymmetric crystal with a monoatomic lattice $|Y|=1, \Phi_{Y}=0$. The following parameters have also been introduced: $X_{r}=\operatorname{Re} \sqrt{\chi_{h} \chi_{\bar{h}}}$ and $X_{i}=\operatorname{Im} \sqrt{\chi_{h} \chi_{\bar{h}}}$. The extinction length in Eq. (18) is defined 

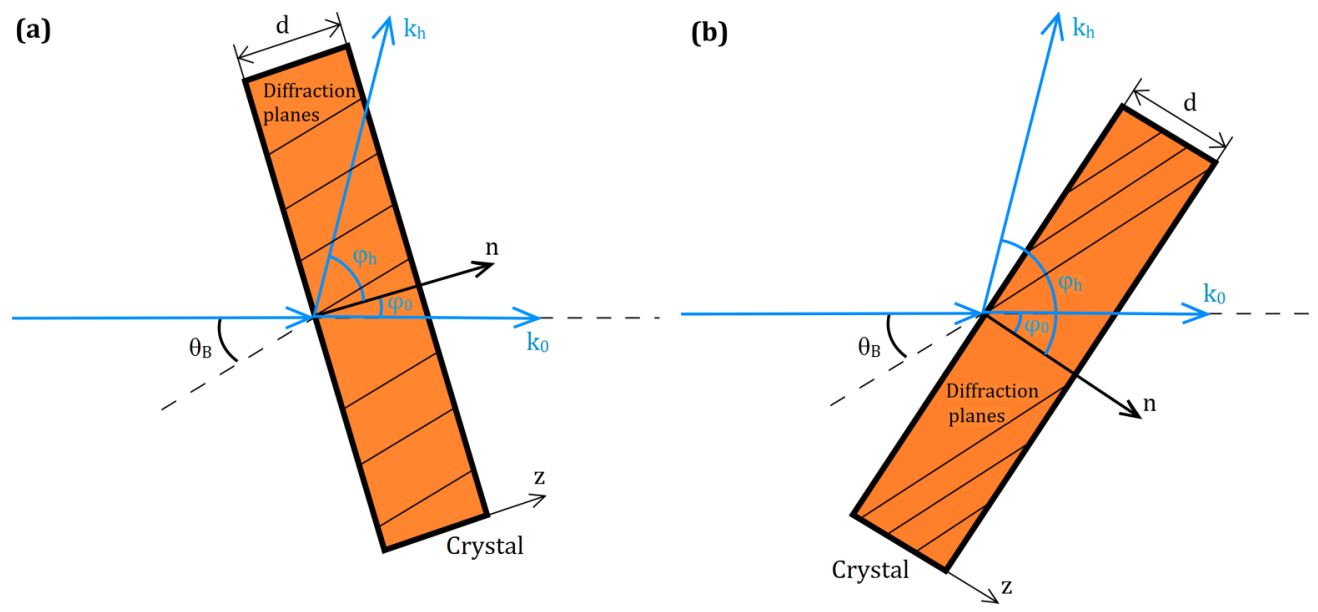

FIG. 2. (a) Diffraction scattering experiment in Laue geometry on a single crystal of the thickness $d$. Here, $\theta_{B}$ is the Bragg angle, $\varphi_{0}$ and $\varphi_{h}$ are the angles between the normal $\mathbf{n}$ to the crystal entrance surface, transmitted $\mathbf{k}_{\mathbf{0}}$ and diffracted $\mathbf{k}_{\mathbf{h}}$ wave vectors, respectively. (b) Diffraction scattering experiment in Bragg geometry on a single crystal of the thickness $d$.

as [24]

$$
L_{e x}=\frac{\lambda \gamma_{0}}{\pi \sqrt{\beta} X_{r}} .
$$

In the case of a perfect thick crystal, $R(z, \Delta \theta)=R_{0}(\Delta \theta)$ and does not depend on the crystal thickness (for details, see Appendix A) and we obtain, for the dynamical phase (18),

$$
\varphi_{\mathrm{dyn}}(d, \Delta \theta)=-\frac{d}{L_{e x}} \operatorname{Re}\left[C_{1} R_{0}(\Delta \theta)\right] .
$$

Below we will present results of dynamical simulations using crystals of different thickness, reflection order, and incident photon energy based on that approach.

\section{Simulations}

We performed full dynamical simulations of the intensity of the diffracted beam and phase of the transmitted beam for gold and silicon crystals of different thickness in Laue geometry. The diffraction scheme was considered asymmetrical in both cases with the incident beam perpendicular to the entrance surface of the crystal as shown in Fig. 1 that corresponds to the choice of angles $\varphi_{0}=0$ and $\varphi_{h}=2 \theta_{B}$ in Fig. 2 . For these simulations, Fourier components of susceptibilities were obtained from Ref. [25] and all scattering parameters are summarized in Table I. We note that for the scattering conditions considered here, the extinction length for Si 111 crystal, $L_{e x}^{S i}=6.1 \mu \mathrm{m}$, was about an order of magnitude larger than for Au 111 crystal, $L_{e x}^{A u}=610 \mathrm{~nm}$.

The reflectivity curves and corresponding phases of the transmitted beam as a function of the rocking angle $\Delta \theta$ were simulated using a full dynamical theory approach. Results of these simulations for $\mathrm{Au}$ and $\mathrm{Si}$ crystals of different thickness from $d=0.2 L_{e x}$ to $d=1.5 L_{e x}$ are presented in Figs. 3 and 4. These simulations show that the angular variation as well as the magnitude of the transmitted wave phase are similar for both crystals for the same ratio of $d / L_{e x}$. The higher the value of this ratio, the stronger is the reflectivity curve and values of the phase modulation. The only difference is the angular range in which these variations of phase are significant. It is much broader in the case of Au crystal and is quite narrow in the case of Si crystal, which can be explained by the difference in the real part of the susceptibility (see Table I). In addition, due to difference in extinction length, the actual thickness of each crystal (Au or $\mathrm{Si}$ ) is significantly different (see Table I).

Simulations performed in the frame of dynamical theory for different reflection orders and incoming photon energies for $\mathrm{Au}$ crystals of thickness $d=300 \mathrm{~nm}$ are presented in Appendix A.

There are also some other common features that can be observed in these simulations. The maximum of the reflectivity curve as well as the angular position of the sign change in the phase are shifted from the exact Bragg position $(\Delta \theta=0)$ to positive values by

$$
\theta_{\text {ref }}=\mp \frac{\chi_{0 r}(1 \pm \beta)}{2 \beta \sin 2 \theta_{B}},
$$

TABLE I. Parameters used in simulations presented in Figs. 3 and 4 for $\mathrm{Au}$ and $\mathrm{Si}$ crystals. In all simulations, $\sigma$ polarization for $\mathrm{x}$ rays was considered.

\begin{tabular}{lcc}
\hline \hline Crystal (reflection) & $\mathrm{Au}(111)$ & $\mathrm{Si}(111)$ \\
\hline Energy $(\mathrm{keV})$ & 8.5 & 8.5 \\
Bragg angle, $\theta_{B}(\mathrm{deg})$ & 18.04 & 13.45 \\
$\gamma_{0}$ & 0 & 0 \\
$\gamma_{h}$ & 0.808 & 0.892 \\
$\chi_{0 r}$ & $-8.31 \times 10^{-5}$ & $-1.35 \times 10^{-5}$ \\
$\chi_{0 i}$ & $6.87 \times 10^{-6}$ & $2.82 \times 10^{-7}$ \\
$\chi_{h r}$ & $-6.83 \times 10^{-5}$ & $-7.16 \times 10^{-6}$ \\
$\chi_{h i}$ & $6.83 \times 10^{-6}$ & $1.97 \times 10^{-7}$ \\
$X_{r}$ & $-6.83 \times 10^{-5}$ & $-7.16 \times 10^{-6}$ \\
$X_{i}$ & $6.83 \times 10^{-6}$ & $1.97 \times 10^{-7}$ \\
$p=-X_{i} / X_{r}$ & 0.10 & 0.027 \\
Extinction length, $L_{e x}(\mathrm{~nm})$ & 610 & 6100 \\
Crystal thickness, $0.2 L_{e x}(\mathrm{~nm})$ & 120 & 1200 \\
Crystal thickness, $0.4 L_{e x}(\mathrm{~nm})$ & 240 & 2400 \\
Crystal thickness, $0.6 L_{e x}(\mathrm{~nm})$ & 370 & 3700 \\
Crystal thickness, $1.5 L_{e x}(\mathrm{~nm})$ & 920 & 9200 \\
\hline \hline
\end{tabular}



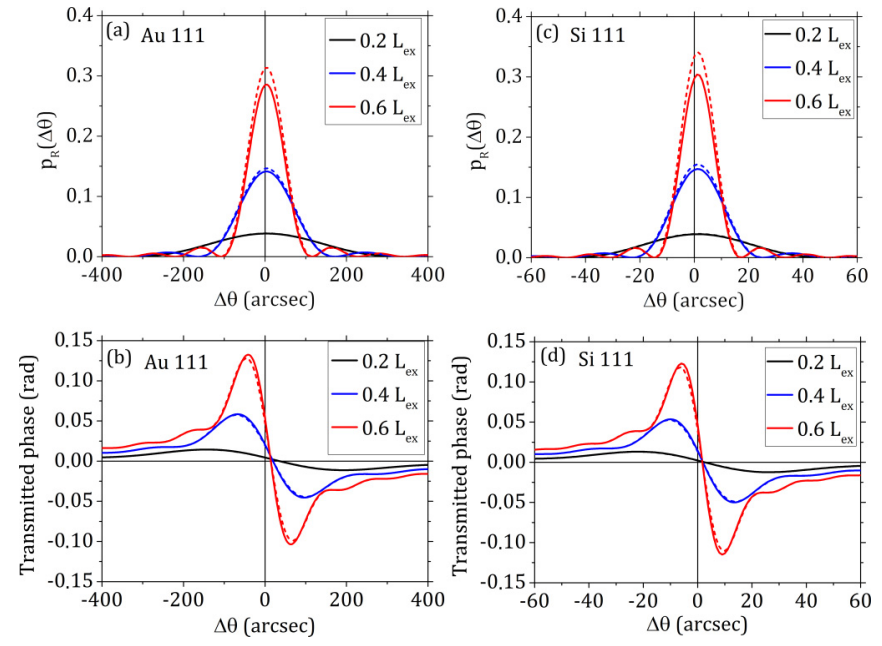

FIG. 3. (a),(c) Reflectivity $p_{R}(\Delta \theta)$ and (b),(d) the phase $\varphi_{\text {dyn }}(\Delta \theta)$ of the transmitted beam in Laue geometry as a function of the rocking angle $\Delta \theta=\theta-\theta_{B}$. Simulations were performed for $\mathrm{Au}$ 111 and Si 111 crystals of different thickness $d=0.2,0.4$, and $0.6 L_{e x}$. Dynamical theory simulations (full lines) and simulations performed in the frame of quasi-kinematical approximation (dashed lines). Parameters of simulations are listed in Table I.

where as before the upper sign corresponds to Bragg diffraction and the lower one to Laue diffraction. This shift due to refraction is well known in dynamical theory [4]. We note that in the case of symmetrical Laue diffraction, when parameter $\beta=1$, this shift due to refraction is zero.

One important feature that we can observe in our simulations is that on the left side of the rocking curve, the phase is positive and goes through its maximum, and on the right side of the rocking curve, it has an opposite behavior. In addition, we can observe that for a thick crystal with $d=1.5 L_{e x}$ negative modulation of the phase is slightly lower than the same positive-valued modulation. To understand the physical reasons for such behavior, we should recall that according to the dynamical theory [4], in the vicinity of the Bragg angle
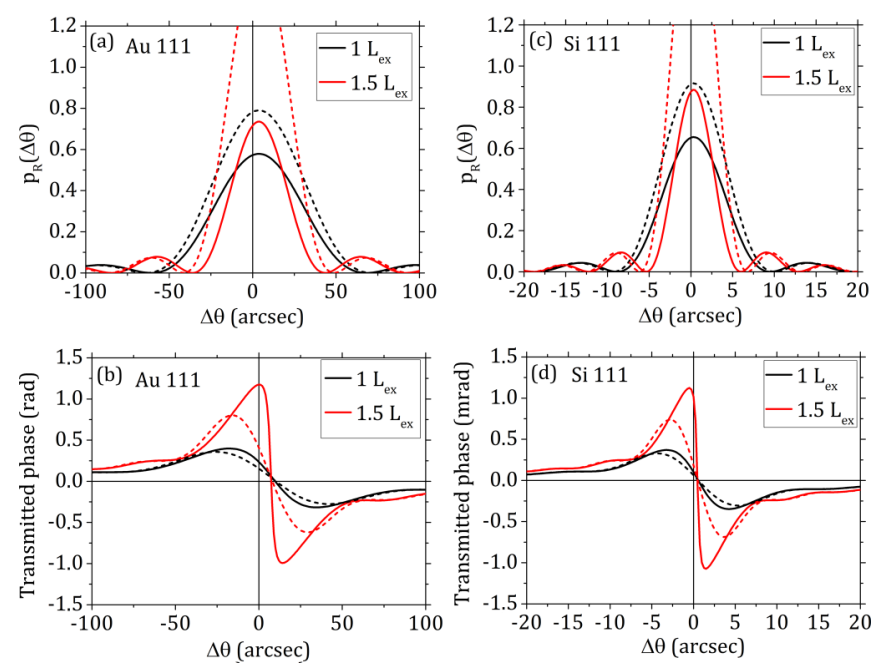

FIG. 4. Same as in Fig. 3 for crystal thickness $d=L_{e x}$ and $1.5 L_{e x}$.


FIG. 5. Angular dependance of the amplitude of the weakly absorbing wave $E_{0}^{(1)}$ (red curve) and strongly absorbing wave $E_{0}^{(2)}$ (black curve) for different crystal thickness (a) $d=L_{e x}$ and (b) $d=10 L_{e x}$. In both cases, simulations were performed for Au 111 reflection and photon energy $8.5 \mathrm{keV}$. The difference in the amplitudes of the waves for the thick crystal is clearly seen.

in Laue geometry, two standing waves are generated: one, weakly absorbing, with its nodes at atomic planes and another one, strongly absorbing, with its antinodes at atomic planes. In thin crystals, both waves contribute with the same strength; however, in a thick crystal, the first wave starts to dominate. Our simulations (see Fig. 5) have shown that on the left side of the rocking curve, the wave field is dominated by the weakly absorbed wave, and on the right side, by the strongly absorbed wave. This also explains the fact that we have on the left side of the rocking curve positive relative values of phase (that means that the wave is accelerated) and we have negative relative values (that means that the wave is retarded) on the right side. This is due to the fact that when the standing wave is with its nodes on the atomic planes, the transmitted wave probes an effectively lower electron density than an averaged electron density. And, on the contrary, when the standing wave is with its antinodes on the atomic planes, the transmitted wave probes an effectively higher electron density than an average electron density.

As mentioned before, these results were obtained using a full dynamical treatment. At the same time, we can easily observe that in the case of thin crystals with $d / L_{e x}<1$, the phase variations of the transmitted beam have similar behavior and are only scaled with the value of the ratio $d / L_{e x}<1$. It would be very useful if a simple analytical expression could be derived to explain the behavior of this phase variation for thin crystals. In the next section, we show how such expression can be obtained from the TT equations (9).

\section{QUASI-KINEMATICAL APPROXIMATION}

We will now consider a thin crystal in Bragg or Laue geometry, such that the transmitted wave does not differ significantly from the incident wave. To characterize the difference between kinematical and dynamical solutions for the transmitted wave, a small parameter

$$
\left|\delta_{\text {dyn }}(z, \Delta \theta)\right| \ll 1
$$

can be introduced as [26]

$$
E_{0}(z, \theta) \approx \exp \left[i \delta_{0} d+i \delta_{\mathrm{dyn}}(z, \Delta \theta)\right]
$$

where parameter $\delta_{0}$ is defined in Eq. (14). Now additional contribution to the phase due to diffraction is given by the real 
part of $\delta_{\text {dyn }}(z, \Delta \theta)$,

$$
\delta \varphi_{\text {dyn }}(z, \Delta \theta)=\operatorname{Re}\left[\delta_{\text {dyn }}(z, \Delta \theta)\right] .
$$

The imaginary part of $\delta_{\mathrm{dyn}}(z, \Delta \theta)$ will give contribution to an interference absorption coefficient $\mu_{\text {in }}(\Delta \theta)$ (for details, see [6]).

By substituting Eq. (24) into TT equations (9) (for details, see Appendix B), it is possible to obtain the following solution for the dynamical contribution to the transmitted beam in the quasi-kinematical approximation:

$$
\begin{aligned}
\delta_{\mathrm{dyn}}(d, \Delta \theta) & =-\frac{C^{2} \chi_{h} \chi_{\bar{h}}(k d)^{2}}{8 \gamma_{0} \gamma_{h}} \frac{1}{\Omega}\left[1-e^{i \Omega}\left(\frac{\sin \Omega}{\Omega}\right)\right], \\
\Omega(\Delta \theta) & =Q(\Delta \theta) d / 2,
\end{aligned}
$$

where $Q(\Delta \theta)=\left(2 / L_{e x}\right)\left[y(\Delta \theta)+i y_{0}\right]$ is a momentum transfer due to angular deviations from the Bragg angle, and dimensionless angular parameters $y(\Delta \theta)$ and $y_{0}$ are defined in Eqs. (A2) and (A3).

Expression (26) for the dynamical contribution to the transmitted beam can also be written in a more compact form by introducing extinction length $L_{e x}$ (20) (see Appendix B),

$$
\delta_{\text {dyn }}(d, \Delta \theta)=-\frac{C_{1}^{2}}{2}\left(\frac{d}{L_{e x}}\right)^{2} \frac{1}{\Omega}\left[1-e^{i \Omega}\left(\frac{\sin \Omega}{\Omega}\right)\right],
$$

where parameter $C_{1}$ is defined after Eq. (19). Taking into account the definition of the phase of the transmitted wave (25), we obtain

$$
\begin{aligned}
& \delta \varphi_{\text {dyn }}(d, \Delta \theta) \\
& =-\frac{C^{2}}{2}\left(\frac{d}{L_{e x}}\right)^{2} \frac{1}{\Omega} \\
& \quad \times\left\{\left(1-p^{2}\right)\left[1-\cos \Omega\left(\frac{\sin \Omega}{\Omega}\right)\right]-2 p \sin \Omega\left(\frac{\sin \Omega}{\Omega}\right)\right\} .
\end{aligned}
$$

It follows from these results that the dynamical contribution to the refraction coefficient is proportional to the product $\chi_{h} \chi_{\bar{h}}$, an expression similar to Eq. (5). Another important feature is that in the quasi-kinematical approximation, the dynamical phase contribution is proportional to the square of the ratio $d / L_{\text {ex }}$ [see Eq. (28)].

In the frame of the same approximations, it is possible to obtain an expression for the amplitude of the diffracted wave (for details, see Appendix B),

$$
E_{h}(d, \Delta \theta)=i E_{h}^{0} e^{i \delta_{h} z} e^{-i \Omega}\left(\frac{\sin \Omega}{\Omega}\right),
$$

where $E_{h}^{0}=C k d \chi_{h} /\left(2 \gamma_{h}\right)$ and parameter $\delta_{h}$ is defined in Eq. (B2) in Appendix B. Substituting this expression in Eqs. (12) and (13), we obtain a well-known expression [19] for the reflectivity in kinematical approximation in Laue or Bragg geometry in a quasi-kinematical approximation,

$$
p_{R}(\Delta \theta)=\frac{\gamma_{h}}{\gamma_{0}}\left|E_{h}^{0}\right|^{2} \frac{\sin ^{2} \Omega}{\Omega^{2}} .
$$

We now consider different asymptotics of the obtained solutions. The dynamical correction in the case of big angular deviations from the exact Bragg angle can be determined from expression (26). For these angles, we can drop off the fast oscillating second term in square brackets in expression (26) and, after substituting the value of the angular parameter $\Omega(\Delta \theta)$ (here we neglect its imaginary part), we obtain

$$
\delta_{\text {dyn }}(d, \Delta \theta) \approx-\frac{C^{2} \chi_{h} \chi_{\bar{h}}(k d)}{4 \gamma_{0} \sin 2 \theta_{B}\left(\Delta \theta-\theta_{\text {ref }}\right)},
$$

where $\theta_{\text {ref }}$ is the angular correction due to refraction (22). Comparison of this expression with the one obtained from the full dynamical theory [see Eq. (5)] shows that they completely coincide.

We can also determine the behavior of the dynamical contribution to the transmitted beam at small values of the angular parameter $\Omega(\Delta \theta)$. From expression (30), it follows that reflectivity of the diffracted wave has its maximum at $\Omega(\Delta \theta)=0$. In the limit of $\Omega(\Delta \theta) \rightarrow 0$, we obtain, for $\delta_{\text {dyn }}(d, \Delta \theta)$ in Eq. (27),

$$
\delta_{\text {dyn }}(d, \Delta \theta) \rightarrow-\frac{C_{1}^{2}}{2}\left(\frac{d}{L_{e x}}\right)^{2}[2 / 3 \Omega-i] .
$$

Substituting the values of parameters $C_{1}$ and $\Omega(\Delta \theta)$, we obtain, for the phase of the transmitted beam,

$$
\begin{aligned}
\delta \varphi_{\text {dyn }}(d, \Delta \theta) \rightarrow & -\frac{C^{2}}{3}\left(\frac{d}{L_{e x}}\right)^{3}\left\{\left(1-p^{2}\right) y(\Delta \theta)\right. \\
& \left.+2 p\left[y_{0}-\frac{3}{2}\left(\frac{L_{e x}}{d}\right)\right]\right\} .
\end{aligned}
$$

Taking into account that $y(\Delta \theta) \sim \Delta \theta$, we see that it is exactly the angular dependence that we observed at small deviations of angular parameter while performing dynamical simulations for thin crystals, as shown in Fig. 3.

Direct comparison of expressions (26) and (29) shows that the dynamical contribution $\delta_{\mathrm{dyn}}(d, \Delta \theta)$ in a quasi-kinematical approximation can be expressed through the amplitude of the diffracted wave as

$$
\delta_{\text {dyn }}(d, \Delta \theta)=-\frac{C_{1}^{2}}{2}\left(\frac{d}{L_{e x}}\right)^{2} \frac{1}{\Omega}\left[1+i \eta(\Delta \theta) E_{h}(d, \Delta \theta)\right],
$$

where the following angular parameter is introduced:

$$
\eta(\Delta \theta)=\frac{2 \gamma_{h}}{C(k d) \chi_{h}} e^{-i \delta_{h} d} e^{2 i \Omega} .
$$

As a result of our analysis [see Eqs. (26), (34), and Appendix C], we see that the phase $\delta \varphi_{\text {dyn }}(d, \Delta \theta)$ modulations grow as a second power of a ratio of a crystal thickness to extinction length $L_{e x}$. At the same time, the quasi-kinematical approximation is valid if condition (23) is satisfied that gives, for the maximum crystal thickness $d_{\max }$,

$$
z \ll d_{\max }=\frac{\sqrt{2}}{\left|C_{1}\right|} L_{e x} .
$$

It follows from Eq. (36) that the quasi-kinematical approximation is valid, in fact, for crystals with the thickness up to extinction length. 


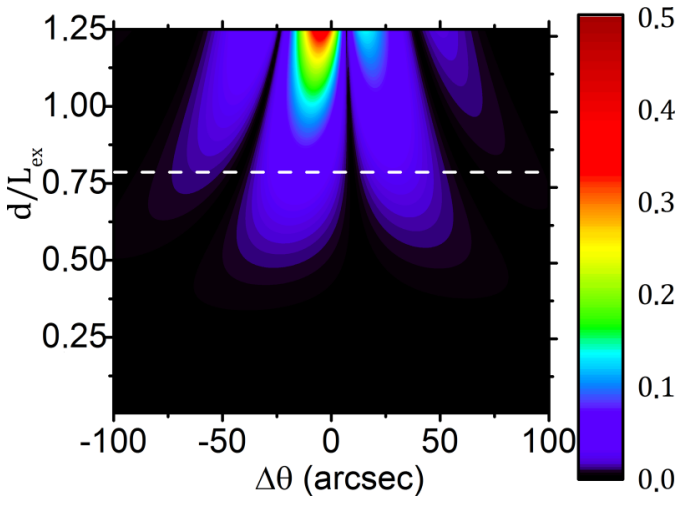

FIG. 6. Comparison between the quasi-kinematical approximation and dynamical theory. Here the relative error $\varepsilon$ in the phase defined in Eq. (37) is calculated using a quasi-kinematical approximation as compared to the dynamical theory as a function of the rocking angle $\Delta \theta$ and relative crystal thickness $d / L_{e x}$. Dashed horizontal line corresponds to $5 \%$ difference in the two phases that appears at the crystal thickness $d \simeq 0.8 L_{e x}$.

We compared the obtained quasi-kinematical result with the exact dynamical solution discussed in the previous section. The dynamical phase correction in the quasi-kinematical approximation given by Eq. (28) was calculated for the same set of parameters as in simulations presented in Figs. 3 and 4. Results of these simulations are shown in these figures by dashed lines. Our results showed that the difference between the quasi-kinematical and dynamical case was less than $3 \%$ for crystal thicknesses up to $0.6 L_{e x}$ (see Fig. 3) and it becomes significant for crystal thicknesses above extinction length (see Fig. 4). It is interesting to note that even for crystals with the thickness $d=L_{e x}$, deviation of the exact dynamical simulation and simulation performed in the frame of the quasi-kinematical approximation is not so strong, though diffracted curves already differ substantially (see Fig. 4). To determine the range of parameters where the quasi-kinematical approach can be safely used, we performed simulations for $\mathrm{Au} 111$ crystal with the thickness varying from zero to $d=1.25 L_{e x}$. We defined an error function $\varepsilon$ between two types of simulations as

$$
\varepsilon=\left|\frac{\delta \varphi_{\mathrm{dyn}}(d, \Delta \theta)-\varphi_{\mathrm{dyn}}(d, \Delta \theta)}{\varphi_{\mathrm{dyn}}(d, \Delta \theta)}\right|,
$$

where $\delta \varphi_{\text {dyn }}(d, \Delta \theta)$ is the dynamical phase contribution in the quasi-kinematical approximation, and $\varphi_{\text {dyn }}(d, \Delta \theta)$ is the same phase simulated with the full dynamical theory. The results of these simulations are presented in Fig. 6. As it follows from these simulations, the quasi-kinematical approach can be safely used with an error less than $5 \%$ up to Au crystal thicknesses $d \simeq 0.8 L_{e x}$. Similar results were also obtained for the Si crystal.

\section{DISCUSSION}

As discussed in Sec. I, the main interest in understanding the properties of the phase of the transmitted beam is the possibility to measure this phase directly in coherence-based experiments such as Bragg coherent $\mathrm{x}$-ray diffractive imaging
(CXDI) and ptychography. To demonstrate the generality of our approach, we will discuss the ptychography experiment depicted in Fig. 1 in more detail.

In a typical ptychographic experiment, the complex amplitude of the exit surface wave can be determined. Specifically, in the case of scattering on a crystal, this exit surface wave can be expressed as [27]

$$
E_{E S W}\left(\mathbf{x}-\mathbf{x}_{\mathbf{j}}\right)=\int G\left(\mathbf{x}-\mathbf{x}^{\prime}\right) E_{\mathrm{in}}\left(\mathbf{x}^{\prime}-\mathbf{x}_{\mathbf{j}}\right) d \mathbf{x}^{\prime},
$$

where $G(\mathbf{x})$ is a Green function of the propagation of x rays in a crystal, $E_{\text {in }}(\mathbf{x})$ is an incident wave field, $\mathbf{x}$ is the transverse coordinate, and $\mathbf{x}_{\mathbf{j}}$ is a position of the incident wave on the sample. Assuming that variations of the incident wave are small on the distances where the Green function is sufficiently changing, we obtain

$$
E_{E S W}\left(\mathbf{x}-\mathbf{x}_{\mathbf{j}}\right)=O(\mathbf{x}) E_{\text {in }}\left(\mathbf{x}-\mathbf{x}_{\mathbf{j}}\right),
$$

where $O(\mathbf{x})$ can be considered as an object function in a typical ptychographic reconstruction. In our case, this object function is defined by the Green function of the crystal through the following relation:

$$
O(\mathbf{x})=\int G\left(\mathbf{x}-\mathbf{x}^{\prime}\right) d \mathbf{x}^{\prime} .
$$

Since ptychography enables us to independently determine both the incident beam $E_{\text {in }}(\mathbf{x})$ (that is often called the probe) and the object function $O(\mathbf{x})$, it also allows us to extend results obtained in this work for plane waves to arbitrary incoming beam shape. The only important condition as stated above is slow variation of the incident beam compared to variations of the Green function. It is known from the dynamical theory [4] that the main variations of the Green function in the crystal are of the size of the Borrmann fan. So, variations of the incident wave should be smaller than the size of the Borrmann fan. Also, importantly, the phase of the transmitted wave is defined completely by the Green function in the form of Eq. (40), which is rather the property of the crystal and not of the incident beam shape. These arguments show that although our results were obtained on the condition of plane-wave illumination in a ptychography experiment, they are valid for a large class of incident beams.

In our simulations, we indirectly used the fact that the crystal lateral size $L$ is large enough to neglect boundary effects. More specifically, it should be larger than the base of the Borrmann fan [4],

$$
L>d \frac{\sin 2 \theta_{B}}{\gamma_{0} \gamma_{h}} .
$$

For example, for Au crystal of $100 \mathrm{~nm}$ thickness used in experiment [18], this condition means that the lateral size of a crystal should be larger than $70 \mathrm{~nm}$. That condition was safely satisfied in this experiment where the lateral size of the crystal was $250 \mathrm{~nm}$.

Additionally, in practical applications, there are some limitations on the beam divergence and energy spread. Assuming that angular divergence of the incoming beam should be much smaller than the angular distance between the two closest extrema on the phase variation curve [see Eq. (C8) 
in Appendix C], we obtain

$$
\delta \theta \ll \frac{\lambda \gamma_{h}}{2 d \sin \left(2 \theta_{B}\right)} .
$$

Using this result and the relation between the wavelength spread and angular divergence $\delta \lambda / \lambda=\delta \theta / \tan \theta_{B}$, we obtain, for the wavelength spread,

$$
\delta \lambda / \lambda \ll \frac{\lambda \gamma_{h}}{4 d \sin ^{2} \theta_{B}} .
$$

In practice, these limitations mean that the angular divergence should be less than $0.8 \mathrm{mrad}$ and the corresponding wavelength spread should be less than $2.6 \times 10^{-3}$ for experiment on a $\mathrm{Au}(111)$ crystal and photon energy $8.5 \mathrm{keV}$. Such conditions can be comfortably achieved at present third-generation synchrotron facilities.

\section{SUMMARY}

We studied the phase variation in the transmitted beam close to Bragg conditions for $\mathrm{Au}$ and $\mathrm{Si}$ crystals of different thickness, reflection, and incoming photon energy using dynamical theory. It was demonstrated that using kinematical theory alone, it was not possible to observe phase variations in the transmitted beam close to the Bragg angle. To perform analysis of scattering in thin crystals, a quasi-kinematical approximation was introduced. A general analytical solution for the phase of the transmitted beam in the whole range of rocking angles was obtained. It was determined that in the quasi-kinematical approximation, the magnitude of the phase variation depends quadratically on the crystal thickness. Possible limitations due to real experimental conditions are also discussed. These findings are of relevance to ptychographic experiments performed on thin crystals where the phase of the transmitted beam can be determined directly from the analysis of the scattered radiation.

\section{ACKNOWLEDGMENTS}

We gratefully acknowledge discussions with I. K. Robinson who directed our attention to this problem. We are also thankful to G. Materlik, M. Hart, and A. Shabalin for fruitful discussions. We acknowledge support of the project and fruitful discussions with $\mathrm{E}$. Weckert and careful reading of the manuscript by D. Novikov. This work was partially supported by the Virtual Institute VH-VI-403 of the Helmholtz Association.

\section{APPENDIX A: DYNAMICAL THEORY TREATMENT}

\section{Scattered amplitude in dynamical theory}

The amplitude $R(z, \Delta \theta)$ (19) can be determined in the general case of the deformed crystal as a solution of a Riccati type of equation (for details, [6]),

$$
\begin{aligned}
\mp i L_{e x} \frac{d R(z, \Delta \theta)}{d z}= & 2\left[-y(\Delta \theta)-i y_{0}+y_{u}\right] R(z, \Delta \theta) \\
& +C_{1}\left[1 \pm R^{2}(z, \Delta \theta)\right],
\end{aligned}
$$

where the upper sign corresponds to Bragg diffraction and the lower one to Laue diffraction. Here the angular deviation from the Bragg position is defined by the dimensionless parameter,

$$
y(\Delta \theta)=\sqrt{\beta} \frac{\sin 2 \theta_{B} \cdot \Delta \theta}{X_{r}} \pm \frac{\chi_{0 r}(1 \pm \beta)}{2 \sqrt{\beta} X_{r}},
$$

and parameters

$$
y_{0}= \pm \frac{\chi_{0 i}(1 \pm \beta)}{2 \sqrt{\beta} X_{r}}, \quad y_{u}(z)= \pm \frac{L_{e x}}{2} \frac{d[\mathbf{h u}(z)]}{d z}
$$

define the attenuation of $\mathrm{x}$ rays due to photoelectric absorption and the shift of the Bragg position caused by deformation in the crystal. Boundary conditions for the amplitude $R(z, \Delta \theta)$ in Eq. (A1) are defined now on one surface. For Bragg geometry, $R(d, \Delta \theta)=0$, and for Laue geometry, $R(0, \Delta \theta)=0$.

In some special cases (for example, constant strain), analytical solutions for the amplitude $R(z, \Delta \theta)$ can be obtained (see, for example, Ref. [6]). In the general case of an arbitrary strain field $\mathbf{u}(z)$, this amplitude can be determined only numerically.

For a perfect thick crystal, the solution of Eq. (A1) gives [6]

$$
R_{0}(\Delta \theta)=\mp \frac{1}{C_{1}}\left\{\left(-y(\Delta \theta)-i y_{0}\right)+\sqrt{\left[y(\Delta \theta)+i y_{0}\right]^{2} \mp C_{1}^{2}}\right\}
$$

where the branch with the positive imaginary part is chosen for the square root and, as before, the upper sign corresponds to Bragg diffraction and the lower one to Laue diffraction.

It is possible to show that in this case of a perfect crystal of arbitrary thickness, an analytical solution for the dynamical amplitude $R(\Delta \theta)$ also exists (see, for details, Ref. [6]). Unfortunately, this solution is complicated for the direct analysis.
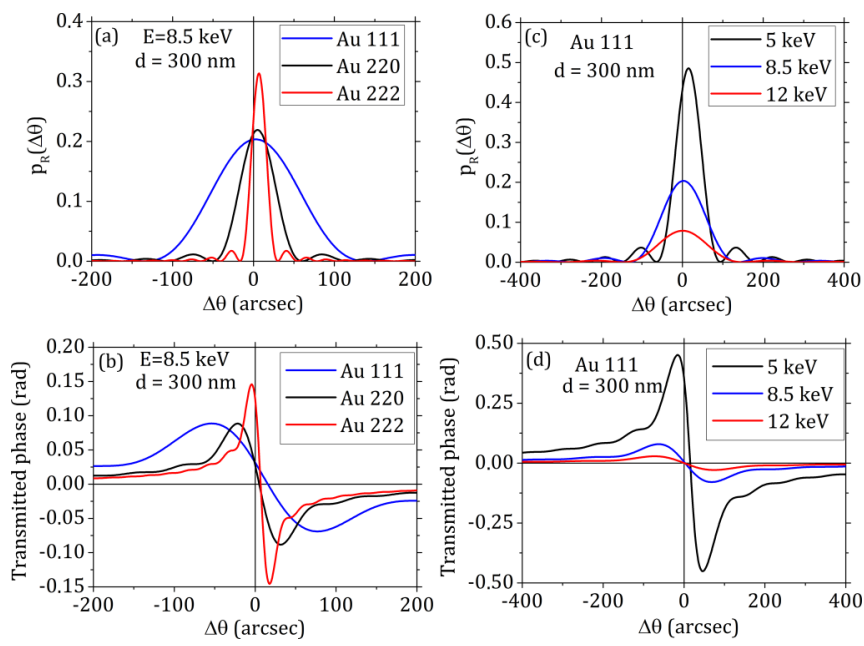

FIG. 7. (a),(c) Reflectivity $p_{R}(\Delta \theta)$ and (b),(d) the phase $\varphi_{\text {dyn }}(\Delta \theta)$ of the transmitted beam in Laue geometry as a function of the rocking angle $\Delta \theta=\theta-\theta_{B}$. Simulations were performed using dynamical theory approach for a Au crystal of thickness $d=300 \mathrm{~nm}$. (a),(b) Results of simulations for the incident photon energy $8.5 \mathrm{keV}$ and different reflection orders 111, 220, and 222 in a Au crystal. (c),(d) Results of simulations for a $\mathrm{Au} 111$ crystal and different incident photon energies of 5, 8.5, and $12 \mathrm{keV}$. Parameters of simulations are listed in Tables II and III. 
TABLE II. Parameters used in simulations presented in Fig. 7(a) and 7(b) for different crystal reflections.

\begin{tabular}{lccc}
\hline \hline Crystal (reflection) & Au (111) & Au (220) & Au (222) \\
\hline Energy (keV) & 8.5 & 8.5 & 8.5 \\
Bragg angle, $\theta_{B}(\mathrm{deg})$ & 18.04 & 30.39 & 38.28 \\
$\gamma_{0}$ & 0 & 0 & 0 \\
$\gamma_{h}$ & 0.808 & 0.488 & 0.232 \\
$\chi_{0 r}$ & $-8.31 \times 10^{-5}$ & $-8.31 \times 10^{-5}$ & $-8.31 \times 10^{-5}$ \\
$\chi_{0 i}$ & $6.87 \times 10^{-6}$ & $6.87 \times 10^{-6}$ & $6.87 \times 10^{-6}$ \\
$\chi_{h r}$ & $-6.83 \times 10^{-5}$ & $-5.63 \times 10^{-5}$ & $-5.04 \times 10^{-5}$ \\
$\chi_{h i}$ & $6.83 \times 10^{-6}$ & $6.75 \times 10^{-6}$ & $6.69 \times 10^{-6}$ \\
$X_{r}$ & $-6.83 \times 10^{-5}$ & $-5.63 \times 10^{-5}$ & $-5.04 \times 10^{-5}$ \\
$X_{i}$ & $6.83 \times 10^{-6}$ & $6.75 \times 10^{-6}$ & $6.69 \times 10^{-6}$ \\
$p=-X_{i} / X_{r}$ & 0.10 & 0.12 & 0.13 \\
Extinction length, $L_{e x}(\mathrm{~nm})$ & 610 & 580 & 440 \\
Crystal thickness, $(\mathrm{nm})$ & 300 & 300 & 300 \\
\hline \hline
\end{tabular}

\section{Phase of the transmitted beam for different reflection orders and incoming photon energies}

Variations of intensity of the diffracted beam and phase of the transmitted beam for different reflection orders as well as for different incoming photon energies for Au crystal are presented in Fig. 7. Here we considered 111, 220, and 222 reflections in $\mathrm{Au}$ crystal and incoming photon energies of 5 , 8.5 , and $12 \mathrm{keV}$. In the first case, the incident photon energy was $8.5 \mathrm{keV}$ for all reflections, and, in the second case, $\mathrm{Au}$ 111 reflection was considered for all energies. In all cases, the crystal thickness was $d=300 \mathrm{~nm}$ and the diffraction scheme was considered the same as shown in Fig. 2. All other scattering parameters are listed in Tables II and III. Here we can see that for higher reflections and lower incident photon energies, the angular range of the phase variations becomes more narrow and variations become stronger, which can be explained by the change of the extinction length (see Table II).

\section{APPENDIX B: DERIVATION OF THE QUASI-KINEMATICAL APPROXIMATION}

To derive an expression for the dynamical correction $\delta_{\text {dyn }}(z, \Delta \theta)$ in transmitted wave $E_{0}(z, \Delta \theta)$ (24) in the quasi-kinematical approximation, we start with the TT equations (9) where we perform the following substitution:

$$
\begin{aligned}
& E_{0}(z)=E_{0}^{\prime}(z) e^{i \delta_{0} z} \text { where } \delta_{0}=\frac{k \chi_{0}}{2 \gamma_{0}}, \\
& E_{h}(z)=E_{h}^{\prime}(z) e^{i \delta_{h} z} \text { where } \delta_{h}=\frac{k\left(\chi_{0}-\alpha\right)}{2 \gamma_{h}},
\end{aligned}
$$

which leads to the following form of the TT equations for the amplitudes $E_{0}^{\prime}(z)$ and $E_{h}^{\prime}(z)$ :

$$
\begin{aligned}
\frac{d E_{0}^{\prime}}{d z} & =\left(\frac{i k}{2 \gamma_{0}}\right) C \chi_{\bar{h}} e^{i \mathbf{h u}(z)} e^{-W(z)} e^{i Q z} E_{h}^{\prime}(z), \\
\frac{d E_{h}^{\prime}}{d z} & =\left(\frac{i k}{2 \gamma_{h}}\right) C \chi_{h} e^{-i \mathbf{h u}(z)} e^{-W(z)} e^{-i Q z} E_{0}^{\prime}(z) .
\end{aligned}
$$

Here, parameter $Q(\Delta \theta)=\left(2 / L_{e x}\right)\left[y(\Delta \theta)+i y_{0}\right]$ is a momentum transfer due to an angular deviation $\Delta \theta$ from the Bragg angle, and dimensionless angular parameters $y(\Delta \theta)$ and $y_{0}$ are defined in Eqs. (A2) and (A3).

Now we consider that in the quasi-kinematical approximation, the transmitted wave $E_{0}^{\prime}(z, \Delta \theta)$ can be presented as

$$
E_{0}^{\prime}(z, \Delta \theta)=e^{i \delta_{\mathrm{dyn}}(z, \Delta \theta)},
$$

TABLE III. Parameters used in simulations presented in Fig. 7(c) and 7(d) for different incoming photon energies.

\begin{tabular}{lccc}
\hline \hline Crystal (reflection) & $\mathrm{Au}(111)$ & $\mathrm{Au}(111)$ & $\mathrm{Au}(111)$ \\
\hline Energy $(\mathrm{keV})$ & 5 & 8.5 & 12 \\
Bragg angle, $\theta_{B}(\mathrm{deg})$ & 31.77 & 18.04 & 12.67 \\
$\gamma_{0}$ & 0 & 0 & 0 \\
$\gamma_{h}$ & 0.44 & $-8.31 \times 10^{-5}$ & 0.904 \\
$\chi_{0 r}$ & $-2.40 \times 10^{-4}$ & $6.87 \times 10^{-6}$ & $-3.806 \times 10^{-5}$ \\
$\chi_{0 i}$ & $4.40 \times 10^{-5}$ & $-6.83 \times 10^{-5}$ & $5.03 \times 10^{-6}$ \\
$\chi_{h r}$ & $-1.97 \times 10^{-4}$ & $6.83 \times 10^{-6}$ & $-3.07 \times 10^{-5}$ \\
$\chi_{h i}$ & $4.37 \times 10^{-5}$ & $-6.83 \times 10^{-5}$ & $5.00 \times 10^{-6}$ \\
$X_{r}$ & $-1.97 \times 10^{-4}$ & $6.83 \times 10^{-6}$ & $-3.07 \times 10^{-5}$ \\
$X_{i}$ & $4.37 \times 10^{-5}$ & 0.10 & $5.00 \times 10^{-6}$ \\
$p=-X_{i} / X_{r}$ & 0.22 & 610 & 0.16 \\
Extinction length, $L_{e x}(\mathrm{~nm})$ & 270 & 300 & 1000 \\
Crystal thickness, $(\mathrm{nm})$ & 300 & 300 \\
\hline \hline
\end{tabular}


where the dynamical correction satisfies the condition $\left|\delta_{\text {dyn }}(z, \Delta \theta)\right| \ll 1$. Substituting this expression for the transmitted wave into the TT equations (B3), keeping derivatives of $\delta_{\text {dyn }}(z, \Delta \theta)$ and approximating $E_{0}^{\prime}(z, \Delta \theta) \simeq 1$ otherwise, we obtain

$$
\begin{aligned}
\frac{d \delta_{\mathrm{dyn}}}{d z} & =\left(\frac{k}{2 \gamma_{0}}\right) C \chi_{\bar{h}} e^{i \mathbf{h u}(z)} e^{-W(z)} e^{i Q z} E_{h}^{\prime}(z), \\
\frac{d E_{h}^{\prime}}{d z} & =\left(\frac{i k}{2 \gamma_{h}}\right) C \chi_{h} e^{-i \mathbf{h u}(z)} e^{-W(z)} e^{-i Q z} .
\end{aligned}
$$

The first equation should be complemented with a boundary condition for a dynamical correction, $\delta_{\mathrm{dyn}}(z=0, \Delta \theta)=0$.

The second equation (B5b) can be easily calculated, leading to the well-known expression for the diffracted wave in kinematical approximation (compare to results in Ref. [6]),

$$
E_{h}^{\prime}(z, \Delta \theta)=i\left(\frac{k C}{2 \gamma_{h}}\right) \chi_{h} \int_{0}^{z} e^{-i \mathbf{h u}(z)} e^{-W(z)} e^{-i Q z} d z .
$$

If the strain field $\mathbf{u}(z)$ and the profile of the static Debye-Waller factor $W(z)$ are known, then integration can be performed using this equation. Unfortunately, these parameters commonly are not known and have to be found using other methods. An especially simple result is obtained in the case of a perfect crystal when $\mathbf{u}(z)=W(z)=0$. In this case, Eq. (B6) reduces to

$$
E_{h}^{\prime}(z, \Delta \theta)=i\left(\frac{k C}{2 \gamma_{h}}\right) \chi_{h} \int_{0}^{z} e^{-i Q z} d z
$$

and its integration gives the well-known expression for the diffracted wave in kinematical approximation for a perfect crystal of thickness $d$,

$$
E_{h}^{\prime}(d, \Delta \theta)=i\left(\frac{C k d}{2 \gamma_{h}}\right) \chi_{h} e^{-i \Omega}\left(\frac{\sin \Omega}{\Omega}\right),
$$

where the dimensionless parameter

$$
\Omega(\Delta \theta)=Q(\Delta \theta) d / 2=\frac{k d}{4} \frac{\alpha \beta+\chi_{0}(1-\beta)}{\gamma_{0}} \approx \frac{k d}{4} \frac{\alpha}{\gamma_{h}}
$$

is introduced.

The dynamical contribution to the transmitted wave, $\delta_{\text {dyn }}(z, \Delta \theta)$, at the exit surface $z=d$ can be obtained by a formal integration of Eq. (B5a),

$$
\delta_{\text {dyn }}(z, \Delta \theta)=\left(\frac{k C}{2 \gamma_{0}}\right) \chi_{\bar{h}} \int_{0}^{d} e^{i \mathbf{h u}(z)} e^{-W(z)} e^{i Q z} E_{h}^{\prime}(z) d z,
$$

where a kinematical solution for the diffracted wave (B6) has to be used. This is a general expression that also includes strain fields in the crystal. It is clear from this expression that the presence of strain can modify the phase of the transmitted beam measured in experiment. Again, we obtain significant simplification if we consider a perfect crystal. In this case, $\mathbf{u}(z)=W(z)=0$, and substituting the expression for the diffracted wave $E_{h}^{\prime}(z)$ (B8) in Eq. (B10) and performing integration, we obtain, finally, for the dynamical correction in the quasi-kinematical approximation,

$$
\delta_{\mathrm{dyn}}(d, \Delta \theta)=-\frac{C^{2} \chi_{h} \chi_{\bar{h}}(k d)^{2}}{8 \gamma_{0} \gamma_{h}} \frac{1}{\Omega}\left[1-e^{i \Omega}\left(\frac{\sin \Omega}{\Omega}\right)\right] .
$$

Now introducing extinction length $L_{e x}$ (20), Eq. (B11) can be written in the following form:

$$
\delta_{\text {dyn }}(d, \Delta \theta)=-\frac{C_{1}^{2}}{2}\left(\frac{d}{L_{e x}}\right)^{2} \frac{1}{\Omega}\left[1-e^{i \Omega}\left(\frac{\sin \Omega}{\Omega}\right)\right],
$$

where parameter $C_{1}$ is introduced after Eq. (19) in the main text, and in the case of a perfect crystal is equal to $C_{1}=$ $C(1-i p)$.

Dynamical phase correction in the transmitted beam $\delta \varphi_{\mathrm{dyn}}(d, \Delta \theta)$ is given by the real part of this expression,

$$
\begin{aligned}
& \delta \varphi_{\text {dyn }}(d, \Delta \theta) \\
& =\operatorname{Re}\left[\delta_{\text {dyn }}(d, \Delta \theta)\right] \\
& =-\frac{C^{2}}{2}\left(\frac{d}{L_{e x}}\right)^{2} \frac{1}{\Omega}\left\{\left(1-p^{2}\right)\left[1-\cos \Omega\left(\frac{\sin \Omega}{\Omega}\right)\right]\right. \\
& \left.\quad-2 p \sin \Omega\left(\frac{\sin \Omega}{\Omega}\right)\right\} .
\end{aligned}
$$

Comparison of expressions (B12) and (B8) shows that the dynamical correction to the transmitted amplitude can also be expressed through the diffracted wave as

$$
\delta_{\mathrm{dyn}}(d, \Delta \theta)=-\frac{C_{1}^{2}}{2}\left(\frac{d}{L_{e x}}\right)^{2} \frac{1}{\Omega}\left[1+i K e^{2 i \Omega} E_{h}^{\prime}(d, \Delta \theta)\right],
$$

where complex parameter $K=2 \gamma_{h} / C(k d) \chi_{h}$ is introduced.

\section{APPENDIX C: ANALYTICAL ANALYSIS OF THE QUASI-KINEMATICAL APPROXIMATION}

The derived expression for $\delta_{D}(26)$ is particularly convenient for obtaining analytical results. First, we should define for which crystal thicknesses the quasi-kinematical approximation is valid. Since the original assumption of the approximation is $\left|\delta_{\mathrm{dyn}}(z, \Delta \theta)\right| \ll 1$, it is necessary to understand when this condition is satisfied. Taking a square modulus of the expression (26), we obtain

$$
\begin{aligned}
\left|\delta_{\mathrm{dyn}}(d, \Delta \theta)\right|^{2}= & \frac{C^{4}\left|\chi_{h} \chi_{\bar{h}}\right|^{2}(k d)^{4}}{64 \gamma_{0}^{2} \gamma_{h}^{2}} \frac{1}{\Omega^{2}}\left|1-e^{i \Omega}\left(\frac{\sin \Omega}{\Omega}\right)\right|^{2} \\
= & \frac{C^{4}\left|\chi_{h} \chi_{\bar{h}}\right|^{2}(k d)^{4}}{64 \gamma_{0}^{2} \gamma_{h}^{2}} \frac{1}{\Omega^{2}}\left(1-\frac{\sin 2 \Omega}{\Omega}\right. \\
& \left.-\frac{\cos 2 \Omega}{2 \Omega^{2}}+\frac{1}{2 \Omega^{2}}\right)
\end{aligned}
$$

where $\Omega$ is approximated to be real according to expression (B9).

In the extrema of $\left|\delta_{\text {dyn }}(\Delta \theta)\right|$, the following condition should be satisfied: $d\left|\delta_{D}\right|^{2} / d \Omega=0$. This is equivalent to the 
following equation:

$$
\left(\cos \Omega-\frac{\sin \Omega}{\Omega}\right)^{2}=0 .
$$

The solutions of Eq. (C2) are $\Omega_{0}=\tan \Omega_{0}$. Substituting this expression into Eq. $(\mathrm{C} 1)$, we obtain, for $\left|\delta_{\mathrm{dyn}}(d)\right|^{2}$ at each extremum,

$$
\left|\delta_{\mathrm{dyn}}(d)\right|^{2}=\frac{C^{4}\left|\chi_{h} \chi_{\bar{h}}\right|^{2}(k d)^{4}}{64 \gamma_{0}^{2} \gamma_{h}^{2}} \frac{1}{1+\Omega_{0}^{2}} .
$$

It follows that the global maximum is present at $\Omega_{0}=0$, and

$$
\left|\delta_{\mathrm{dyn}}(d)\right|_{\Omega=0}=\frac{C^{2}\left|\chi_{h} \chi_{\bar{h}}\right|(k d)^{2}}{8 \gamma_{0} \gamma_{h}} .
$$

Substituting expression (C4) into $\left|\delta_{\text {dyn }}(z, \Delta \theta)\right| \ll 1$, we obtain

$$
d \ll \frac{\sqrt{2}}{\left|C_{1}\right|} L_{e x} .
$$

Therefore, as one could expect, the near-kinematical approximation is valid for small thicknesses.

Next, we want to find the extrema of the quasi-kinematical expression (28) for the phase. Substituting expression (28) into the condition $d\left(\delta \varphi_{\text {dyn }}\right) / d \Omega=0$, we immediately obtain

$$
\left[\left(1-p^{2}\right) \cos \Omega+2 p \sin \Omega\right]\left(\frac{\sin \Omega}{\Omega}-\cos \Omega\right)=0 .
$$

The factor on the right gives local extrema for the phase, located at the same points as the extrema of $\left|\delta_{\text {dyn }}(\Delta \theta)\right|$. The factor on the left gives

$$
\tan \Omega=\frac{p^{2}-1}{2 p}=\tan \left(\frac{1}{\pi}-\varphi_{\chi_{h} \chi_{\bar{h}}}\right),
$$

where $\varphi_{\chi_{h} \chi_{\bar{h}}}$ is the complex phase of $\chi_{h} \chi_{\bar{h}}$. We are interested in two extrema closest to $\Omega=0$, which are

$$
\Omega_{0}=\left(\frac{\pi}{2}-\varphi_{\chi_{h} \chi_{\bar{h}}}\right) \bmod \pi,\left(\frac{\pi}{2}-\varphi_{\chi_{h} \chi_{\bar{h}}}\right) \bmod \pi-\pi,
$$

where $x$ mod $y$ represents the non-negative remainder when dividing $x$ by $y$.

Substituting those $\Omega$ values into Eq. (28), we get the extremum value

$$
\delta \varphi_{\mathrm{dyn}}(d)=-\frac{C^{2}}{2}\left(\frac{d}{L_{e x}}\right)^{2}\left(1-p^{2}\right) \frac{1}{\Omega_{0}} .
$$

For reflections considered in this work, $\varphi_{\chi_{h} \chi_{\bar{h}}}=0$ and $\Omega= \pm \pi / 2$. However, in crystals without central symmetry, $\varphi_{\chi_{h} \chi_{\bar{h}}} \neq 0$. Equation (C9) shows that the phase $\delta \varphi_{\text {dyn }}(d, \Delta \theta)$ modulations indeed grow as a second power of a ratio of a crystal thickness to extinction length $L_{e x}$.
[1] J. Als-Nielsen and D. McMorrow, Elements of Modern X-ray Physics, 2nd ed. (Wiley, Singapore, 2011).

[2] A. Snigirev, V. Kohn, I. Snigireva, and B. Lengeler, Nature (London) 384, 49 (1996).

[3] S. W. Wilkins, T. E. Gureyev, D. Gao, A. Pogany, and A. W. Stevenson, Nature (London) 384, 335 (1996).

[4] A. Authier, Dynamical Theory of X-ray Diffraction, 2nd ed. (Oxford University Press, Oxford, 2003).

[5] B. Batterman and H. Cole, Rev. Mod. Phys. 36, 681 (1964).

[6] I. Vartanyants and M. Kovalchuk, Rep. Prog. Phys. 64, 1009 (2001).

[7] The X-ray Standing Wave Technique. Principles and Applications, edited by J. Zegenhagen and A. Kazimirov (World Scientific, Singapore, 2013).

[8] V. E. Dmitrienko and V. A. Belyakov, Sov. Tech. Phys. Lett. 6, 621 (1980)

[9] V. A. Belyakov and V. E. Dmitrienko, Sov. Phys. Usp. 32, 697 (1989).

[10] K. Hirano, K. Izumi, T. Ishikawa, S. Annaka, and S. Kikuta, Jpn. J. Appl. Phys. Lett. 30, L407 (1991).

[11] C. Giles, C. Malgrange, J. Goulon, F. de Bergevin, C. Vettier, E. Dartyge, A. Fontaine, C. Giorgetti, and S. Pizzini, J. Appl. Crystallogr. 27, 232 (1994).

[12] G. Geloni, V. Kocharyan, and E. Saldin, J. Mod. Opt. 58, 1391 (2011).

[13] J. Amann, W. Berg, V. Blank, F.-J. Decker, Y. Ding, P. Emma, Y. Feng, J. Frisch, D. Fritz, J. Hastings et al., Nat. Photon. 6, 693 (2012)
[14] K. Hirano and A. Momose, Phys. Rev. Lett. 76, 3735 (1996).

[15] U. Bonse and M. Hart, Appl. Phys. Lett. 6, 155 (1965).

[16] J. M. Rodenburg, A. C. Hurst, A. G. Cullis, B. R. Dobson, F. Pfeiffer, O. Bunk, C. David, K. Jefimovs, and I. Johnson, Phys. Rev. Lett. 98, 034801 (2007).

[17] P. Thibault, M. Dierolf, A. Menzel, O. Bunk, C. David, and F. Pfeiffer, Science 321, 379 (2008).

[18] M. Civita, A. Diaz, R. J. Bean, A. Shabalin, O. Gorobtsov, I. A. Vartaniants, and I. K. Robinson (unpublished).

[19] W. Zachariazen, Theory of X-ray Diffraction in Crystals (Dover, New York, 1967).

[20] Note the difference by a factor of two between this expression and the one given in the book [4].

[21] S. Takagi, J. Phys. Soc. Jpn. 26, 1239 (1969).

[22] D. Taupin, Acta Crystallogr. 23, 25 (1967).

[23] We should note that strictly speaking in the case of kinematical scattering the transmitted amplitude is equal to unity. Here we include in the transmitted amplitude refraction and normal absorption but neglect dynamical effects of multiple scattering.

[24] It should be noted that our choice of extinction length differs from that commonly used [4] by the factor $\pi$.

[25] S. A. Stepanov, http://sergey.gmca.aps.anl.gov/x0h.html (unpublished).

[26] Compare this expression with the one for the transmitted beam in kinematical approximation (15).

[27] A. Afanasév and V. Kohn, Acta Crystallogr. Sect. A 27, 421 (1971). 